\title{
Are multidisciplinary teams in secondary care cost-effective? A systematic review of the literature
}

K Melissa Ke ${ }^{1 *}$, Jane M Blazeby ${ }^{2,3}$, Sean Strong ${ }^{2}$, Fran E Carroll ${ }^{2}$, Andy R Ness ${ }^{1}$ and William Hollingworth ${ }^{2}$

\begin{abstract}
Objective: To investigate the cost effectiveness of management of patients within the context of a multidisciplinary team (MDT) meeting in cancer and non-cancer teams in secondary care.
\end{abstract}

Design: Systematic review.

Data sources: EMBASE, MEDLINE, NHS EED, CINAHL, EconLit, Cochrane Library, and NHS HMIC.

Eligibility criteria for selecting studies: Randomised controlled trials (RCTs), cohort, case-control, before and after and cross-sectional study designs including an economic evaluation of management decisions made in any disease in secondary care within the context of an MDT meeting.

Data extraction: Two independent reviewers extracted data and assessed methodological quality using the Consensus on Health Economic Criteria (CHEC-list). MDTs were defined by evidence of two characteristics: decision making requiring a minimum of two disciplines; and regular meetings to discuss diagnosis, treatment and/or patient management, occurring at a physical location or by teleconferencing. Studies that reported on the costs of administering, preparing for, and attending MDT meetings and/or the subsequent direct medical costs of care, non-medical costs, or indirect costs, and any health outcomes that were relevant to the disease being investigated were included and classified as cancer or non-cancer MDTs.

Results: Fifteen studies (11 RCTs in non-cancer care, 2 cohort studies in cancer and non-cancer care, and 2 before and after studies in cancer and non cancer care) were identified, all with a high risk of bias. Twelve papers reported the frequency of meetings which varied from daily to three monthly and all reported the number of disciplines included (mean 5, range 2 to 9). The results from all studies showed mixed effects; a high degree of heterogeneity prevented a meta-analysis of findings; and none of the studies reported how the potential savings of MDT working may offset the costs of administering, preparing for, and attending MDT meetings.

Conclusions: Current evidence is insufficient to determine whether MDT working is cost-effective or not in secondary care. Further studies aimed at understanding the key aspects of MDT working that lead to cost-effective cancer and non-cancer care are required.

Keywords: Multidisciplinary teams, Cost-effective, Secondary care

\footnotetext{
* Correspondence: Melissa.Ke@bristol.ac.uk

${ }^{1}$ School of Oral and Dental Sciences, University of Bristol, Lower Maudlin Street, Bristol BS1 2LY, UK

Full list of author information is available at the end of the article
} 


\section{Introduction}

Multi-disciplinary teams (MDTs) are an integrated approach to healthcare in which medical and allied health professionals consider relevant treatment options and collaboratively develop an individual treatment plan for patients [1,2]. Despite some scepticism about the effectiveness of MDT working in health care [2-4], the concept of MDT working is now widely accepted [5] and considered to be good practice in many parts of the world for the successful management of chronic disease [6-8]. Teamwork is recommended because of increasing complexity in patient management and subspecialisation of health professionals, and because of changes in working patterns in the health care sector with reduced hours of work [9].

Areas where MDT working is now commonly adopted in secondary care include care of the elderly, heart failure, stroke, mental health, and critical care. The area that has seen the most increase in MDT working, however, is in oncology where MDT working has been widely recommended including in North America [10], Australia [11,12], and continental Europe [13,14]. Indeed it is now mandated as part of the NHS Cancer Plan in the UK [6]. Studies have shown that MDT working in cancer care is associated with decreased time from diagnosis to treatment [15], more accurate pathological staging of disease [16], increased number of patients treated with radical intent $[17,18]$, improved survival [19], and greater patient satisfaction [20]. However, these studies are largely retrospective, and there is a lack of well designed randomised controlled trials and prospective studies in this field [1,21-23]. There is also a need to consider the costs of administering, preparing for, and attending MDT meetings in any evaluation.

MDTs demand considerable organisation, management infrastructure and funding to ensure that the correct personnel are present, that the relevant patient details are collated, and that the pathological and radiological materials are reviewed by the appropriate specialists before and during the meeting $[1,24,25]$. Indeed, it has been shown that if critical information is absent at the MDT meeting, decisions may need to change following the meeting and this can delay the start of treatment [26]. In the UK enormous investment has been made to ensure the functioning of cancer MDT meetings and it is estimated to cost the National Health Service (NHS) around $£ 50$ million a year for preparation and a similar amount for attendance time [23]. Whether this substantial investment is justified, however, is unknown and has not been previously considered. The aim, therefore, of this paper was to systematically summarise economic studies of MDT working in secondary cancer and non-cancer care.

\section{Methods}

\section{Criteria for considering studies for this review Types of studies}

We reviewed economic evaluations of MDT working compared to no MDT in the provision of secondary care which includes hospital-based and community-based care (Table 1). The types of study designs eligible for inclusion were randomised controlled trials (RCTs), cohort, case-control, before and after, cross-sectional, and decision analysis modelling studies.

\section{Types of participants}

We included studies which enrolled people with any disease type, receiving secondary care and we classified these as cancer and non-cancer.

\section{Types of interventions}

MDTs were defined by evidence of both of the following characteristics:

1. Team members from a minimum of two disciplines making decisions;

2. Regular team meetings to discuss diagnosis, treatment and/or patient management, occurring at a physical location or by teleconferencing.

Where team members came from different grades within one discipline, this was considered to represent a single discipline (e.g. a consultant surgeon and a specialist surgical registrar were counted as one discipline).

Studies that evaluated MDT decision making at the patient bedside during ward rounds were excluded because we felt that they were substantially different from the type of meeting room based MDT work advocated in recent policy initiatives (e.g. for cancer services) [6,27]. The key differences between the two are that meeting room based MDTs require a coordinator, and include a systematic review of information about the patient without the patients' input or interruptions. If a paper did not state whether the MDT meeting was ward-based or meeting room based, this was inferred from the description of the meeting.

\section{Types of outcome measures}

Full economic evaluations (i.e. cost-effectiveness analysis, cost-utility analysis, cost-benefit analysis and costconsequences analysis) [28] were considered for inclusion.

\section{Types of costs}

We included studies that reported on the costs of supporting an MDT meeting or the subsequent direct medical costs of care, non-medical costs (e.g. patient travel), or indirect costs (e.g. productivity losses). The costs of supporting an MDT typically include the costs of 
Table 1 Inclusion and exclusion criteria for systematic review on cost-effectiveness of MDT working

\begin{tabular}{|c|c|}
\hline Inclusion criteria & Exclusion criteria \\
\hline Studies comparing MDT care with no MDT care & Studies that are not comparative i.e. focus on MDT care only \\
\hline Secondary care services i.e. hospital-based or community-based & Primary care \\
\hline $\begin{array}{l}\text { Study design - RCT, cohort, case-control, before and after, cross-sectional studies, } \\
\text { or modelling studies }\end{array}$ & Ecological studies, case reports \\
\hline Applied study (i.e. studies generating primary data or modelling of secondary data) & $\begin{array}{l}\text { Methodological and general articles, expert opinion, letters } \\
\text { and abstracts }\end{array}$ \\
\hline \multicolumn{2}{|l|}{ Population - persons diagnosed with any diseases } \\
\hline \multicolumn{2}{|l|}{ Study setting - any country } \\
\hline MDTs are defined as: & Multidisciplinary ward rounds \\
\hline \multicolumn{2}{|l|}{ a) Team members from a minimum of two disciplines making decisions; and } \\
\hline \multicolumn{2}{|l|}{$\begin{array}{l}\text { b) Regular team meetings to discuss diagnosis, treatment and/or patient management, } \\
\text { occurring at a physical location or by tele-conferencing }\end{array}$} \\
\hline \multicolumn{2}{|l|}{ Outcomes - health outcomes which are relevant to the disease being investigated } \\
\hline \multicolumn{2}{|l|}{$\begin{array}{l}\text { Costs - average costs of organising MDT meetings, average cost per patient treated, } \\
\text { or incremental cost effectiveness ratios }\end{array}$} \\
\hline Journal articles, grey literature & Books \\
\hline English language & Foreign languages \\
\hline
\end{tabular}

team members preparing and attending MDT meetings; the latter could include travel expenses, or costs of teleconferencing, as well as administrative support provided by a team coordinator.

\section{Search methods for identification of studies}

We searched the databases EMBASE and MEDLINE (both via the Ovid interface), NHS EED, CINAHL, EconLit, Cochrane Library, and NHS HMIC for relevant publications published from the date of inception of each database to December 2011. Grey literature was identified through the NHS HMIC database as well as based on specialised knowledge of one of the authors (JMB). The search strategy consisted of combinations of free text and $\mathrm{MeSH}$ terms related to the economic evaluation of an MDT (see Appendix 1). The search included journal articles and grey literature published in the English language. Reference lists from included studies and other relevant publications, including reviews, were manually checked for citations missed by the electronic search.

\section{Data collection and analysis Selection of studies}

Initially, one author (KMK) selected reports fulfilling the first inclusion criterion (see Table 1) of this review based on title and abstract. Full articles of possibly relevant studies were retrieved for more detailed evaluation. A final selection of included studies was decided by discussion with the whole team.

\section{Data extraction}

A data extraction template, developed using the guidelines provided by the Centre for Reviews and Dissemination [29], was used to extract the following data alongside the critical appraisal of original studies: country of investigation; objective of the study; study intervention and comparator; study design and setting; target population characteristics; sources and quality of clinical data, if applicable; sources and quality of cost data; methods for dealing with uncertainty; and study results. The following data detailing the characteristics of MDT meetings were also extracted: frequency of meetings, number of disciplines contributing, meeting venue, description of leadership style, method of dissemination of decision, and administrative support. Two authors (KMK, SS) independently extracted data. All discrepancies were reviewed and consensus achieved by discussion.

Due to the varied nature of the studies, we provide a narrative summary of the study results for our review rather than a formal meta-analysis of results.

\section{Methodological quality assessment}

The methodological quality of the included studies was assessed using the Consensus on Health Economic Criteria (CHEC-list), a 19-item assessment tool [30]. The CHEC-list represents a minimum set of methodological criteria that address aspects of the internal and external validity of individual economic evaluation studies. For each criterion, a study received a 'yes' if it had sufficiently addressed that aspect of the study, and a 'no' if it had not. Two authors (KMK, FEC) independently evaluated the 
methodological quality of included studies. Both piloted the use of the CHEC-list. Disagreement was resolved by discussion.

The six CHEC-list criteria that are regarded as most important in assessing risk of bias are appropriate choice of an time horizon, measurement of costs, valuation of costs, measurement of outcomes, valuation of outcomes, and discounting of future costs and outcomes [31]. Therefore, the risk of bias among the included studies would be high if the number of studies that fulfilled these six criteria is low.

\section{Results}

\section{Overview}

Our electronic literature search identified 1,788 articles. After a detailed review of 63 articles, 15 were included in the final analysis (Figure 1) [32-46]. Eleven were based on RCTs, [32,34,36,38-41,43-46] most commonly performed in diseases of older people $[34,39,40,44]$ (Table 2). Only two studies evaluated MDT working in cancer care [35,37], and none were conducted in the UK. The majority of studies only included costs borne by the health care payer. No studies estimated the full cost of administering, preparing for and attending an MDT meeting.

\section{Methodological quality assessment}

Only four of the nineteen criteria (i.e. research question posed in answerable form, appropriate economic study design, appropriate time horizon, and identification of important and relevant outcomes) were met by all the studies (Table 3). The choice of an appropriate time horizon is one of the six CHEC-list criteria that are regarded as important in assessing risk of bias [31]. None of the other five criteria were fulfilled by all the studies. In particular, the criteria of appropriate valuation of costs and appropriate discounting of future costs were met by very few studies - four and two respectively. The reasons for a high number of studies not meeting the criterion of appropriate valuation of costs were either a lack of information on the method of valuation, the reference year, or the use of a top-down approach in the apportionment of costs ${ }^{\mathrm{a}}$. There were four studies in the review that should have discounted future costs and outcomes because their analytic horizon was more than a year. However, only two $[42,43]$ stated that discounting was undertaken in the analysis. Therefore, given that five of the six criteria that had an impact on the assessment of risk of bias were not met by all studies, there is a relatively high risk of bias in the results of the reviewed studies.

\section{Characteristics of MDT meetings}

The amount of detail provided about MDT meetings varied. Twelve papers reported the frequency of meetings which varied from daily to once every three months (Table 4). The average number of disciplines in each MDT was 5 ranging from 2 to 9 . Only one study stated the venue of the MDT meeting [44]. Five of the studies gave details of who led the meetings. No information was provided in any study about the amount of administrative

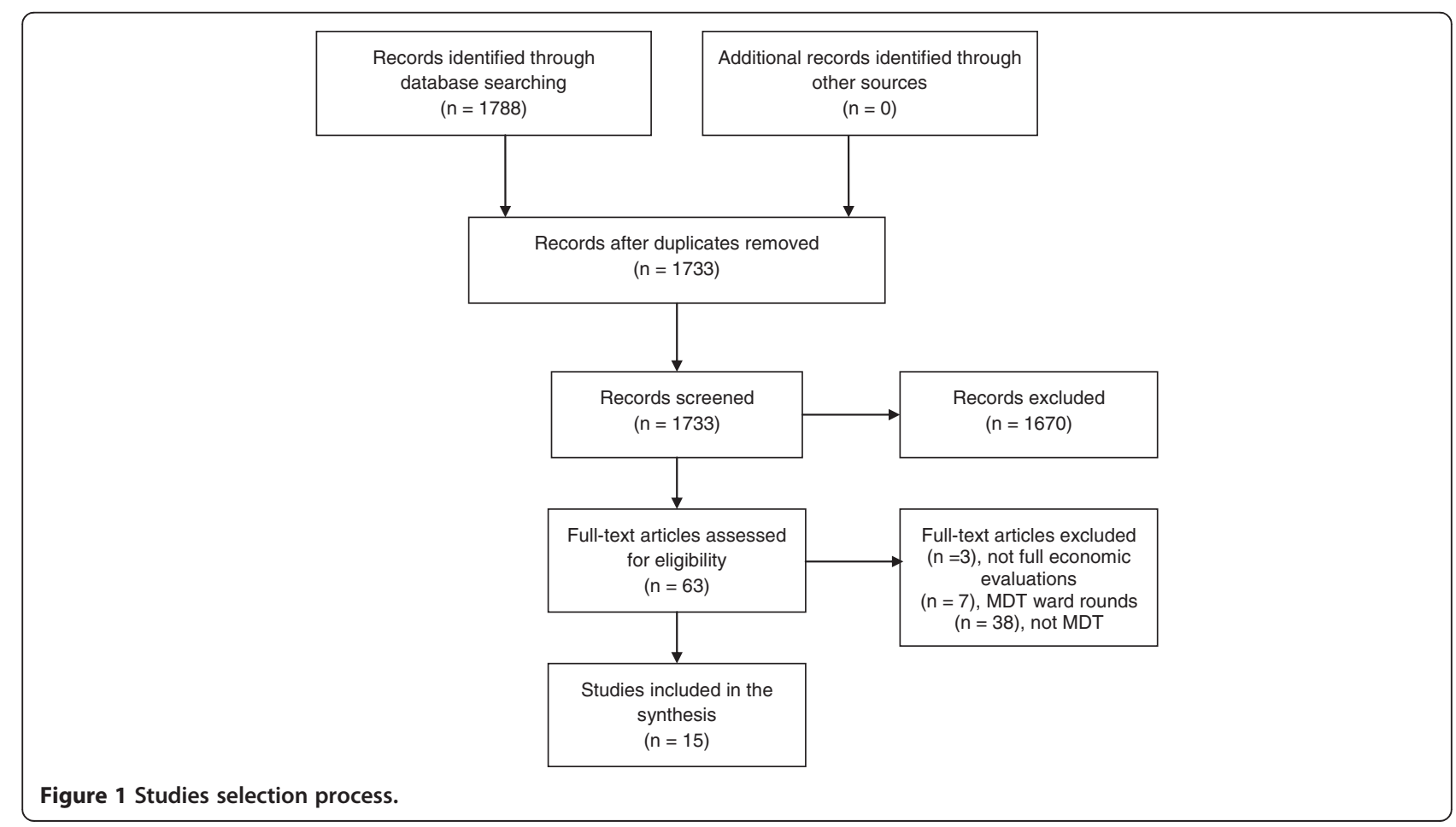




\begin{tabular}{|c|c|}
\hline Characteristics & Number of studies \\
\hline \multicolumn{2}{|l|}{ Study country } \\
\hline USA & 8 \\
\hline The Netherlands & 2 \\
\hline Japan & 2 \\
\hline Ireland & 1 \\
\hline Italy & 1 \\
\hline Sweden & 1 \\
\hline \multicolumn{2}{|l|}{ Study design } \\
\hline $\mathrm{RCT}$ & 11 \\
\hline Cohort & 2 \\
\hline Before and After & 2 \\
\hline \multicolumn{2}{|l|}{ Type of economic evaluation } \\
\hline Cost-consequences analysis & 12 \\
\hline Cost-utility analysis & 2 \\
\hline Cost-benefit analysis & 1 \\
\hline \multicolumn{2}{|l|}{ Disease type } \\
\hline Geriatrics & 4 \\
\hline Cancer & 2 \\
\hline Heart failure & 2 \\
\hline Terminal/critical illness & 2 \\
\hline Various & 2 \\
\hline Mental health & 1 \\
\hline Rheumatoid arthritis & 1 \\
\hline Stroke & 1 \\
\hline \multicolumn{2}{|l|}{ Study perspective } \\
\hline Health care payer & 12 \\
\hline Societal & 3 \\
\hline \multicolumn{2}{|l|}{ Care setting* } \\
\hline Inpatient & 8 \\
\hline Outpatient & 8 \\
\hline \multicolumn{2}{|l|}{ Type of decisions } \\
\hline Treatment/care plan & 11 \\
\hline Diagnosis and treatment/care plan & 4 \\
\hline
\end{tabular}

* 1 study had both inpatient and outpatient care.

support required for an MDT meeting. Finally, 11 reported how the MDT decisions were disseminated.

\section{RCTs assessing cost-effectiveness of MDTs in non-cancer care}

There were 11 RCTs that reported full economic evaluations of MDT care (Table 5). Four evaluated geriatric care, and the results were mixed. One RCT found that MDT directed care resulted in lower costs as well as improvement in some outcome measures. This study
Table 3 Number of studies fulfilling each Consensus on Health Economic Criteria (CHEC-list) quality criterion

\begin{tabular}{|c|c|}
\hline CHEC-list quality criteria & $\begin{array}{l}\text { Number of studies } \\
\text { fulfilling criterion }\end{array}$ \\
\hline 1. Is the study population clearly described? & 12 \\
\hline 2. Are competing alternatives clearly described? & 14 \\
\hline $\begin{array}{l}\text { 3. Is a well-defined research question posed in } \\
\text { answerable form? }\end{array}$ & 15 \\
\hline $\begin{array}{l}\text { 4. Is the economic study design appropriate to } \\
\text { the stated objective? }\end{array}$ & 15 \\
\hline $\begin{array}{l}\text { 5. Is the chosen time horizon appropriate in order } \\
\text { to include relevant costs and consequences? }\end{array}$ & 15 \\
\hline 6. Is the actual perspective chosen appropriate? & 3 \\
\hline $\begin{array}{l}\text { 7. Are all important and relevant costs for each } \\
\text { alternative identified? }\end{array}$ & 0 \\
\hline $\begin{array}{l}\text { 8. Are all costs measured appropriately in physical } \\
\text { units? }\end{array}$ & 11 \\
\hline 9. Are costs valued appropriately? & 4 \\
\hline $\begin{array}{l}\text { 10. Are all important and relevant outcomes for } \\
\text { each alternative identified? }\end{array}$ & 15 \\
\hline 11. Are all outcomes measured appropriately? & 13 \\
\hline 12. Are outcomes valued appropriately? & 13 \\
\hline $\begin{array}{l}\text { 13. Is an incremental analysis of costs and } \\
\text { outcomes of alternatives performed? }\end{array}$ & 3 \\
\hline $\begin{array}{l}\text { 14. Are all future costs and outcomes discounted } \\
\text { appropriately? }\end{array}$ & 2 \\
\hline $\begin{array}{l}\text { 15. Are all important variables, whose values are } \\
\text { uncertain, appropriately subjected to sensitivity } \\
\text { analysis? }\end{array}$ & 3 \\
\hline $\begin{array}{l}\text { 16. Do the conclusions follow from the data } \\
\text { reported? }\end{array}$ & 9 \\
\hline $\begin{array}{l}\text { 17. Does the study discuss the generalizability of } \\
\text { the results to other settings and patient/client } \\
\text { groups? }\end{array}$ & 2 \\
\hline $\begin{array}{l}\text { 18. Does the article indicate there is no potential } \\
\text { conflict of interest of study researcher(s) and } \\
\text { funder(s)? }\end{array}$ & 4 \\
\hline $\begin{array}{l}\text { 19. Are ethical and distributional issues discussed } \\
\text { appropriately? }\end{array}$ & 0 \\
\hline
\end{tabular}

included two sites, and it was found that for one of the sites, total costs were lower by approximately $15 \%$, and there were fewer complications during hospitalisation (between 3 to 12 fewer for different types of complications) [34]. However, two other RCTs of geriatric care concluded that there was no evidence of differences in costs or outcomes between MDT and no MDT care $[39,44]$. The fourth RCT in geriatric care showed that MDT care was associated with higher costs because the cost of implementing MDT care ${ }^{b}$ for 6 months offset the reduction in medication costs, but there was no evidence of differences in functional status and survival between the two groups [40].

Similarly, there were inconsistent results from two RCTs of MDT care for patients suffering from heart 
Table 4 Characteristics of MDT meeting

\begin{tabular}{|c|c|c|c|c|c|c|}
\hline Study & $\begin{array}{l}\text { Frequency of } \\
\text { meeting }\end{array}$ & $\begin{array}{l}\text { Number of } \\
\text { disciplines }\end{array}$ & $\begin{array}{l}\text { Meeting } \\
\text { venue }\end{array}$ & Leadership & $\begin{array}{l}\text { Administration } \\
\text { support }\end{array}$ & $\begin{array}{c}\text { Dissemination of } \\
\text { decision }\end{array}$ \\
\hline Collard et al., 1985 & Twice a week & 6 & Not stated & Not stated & Not stated & Not stated \\
\hline Williams et al., 1987 & Daily & 6 & Outpatient clinic & Not stated & Not stated & Care plan \\
\hline Timpka et al., 1997 & Weekly & 3 & Not stated & Physicians & Not stated & Not stated \\
\hline Fader et al., 1999 & Biweekly & 9 & Not stated & Not stated & Not stated & Treatment plan \\
\hline Kominski et al., 2001 & $\begin{array}{c}\text { At least once every } \\
3 \text { months }\end{array}$ & 8 & Not stated & Not stated & Not stated & Treatment plan \\
\hline Capomolla et al., 2002 & Not stated & 6 & Not stated & Not stated & Not stated & A report \\
\hline Kasper et al., 2002 & Weekly & 3 & Not stated & Not stated & Not stated & Treatment plan \\
\hline Carling et al., 2003 & Not stated & 2 & Not stated & Infectious disease physician & Not stated & $\begin{array}{l}\text { Pro forma placed in } \\
\text { front of patient's chart }\end{array}$ \\
\hline Van Den Hout et al., 2003 & Weekly & 5 & Not stated & Not stated & Not stated & Not stated \\
\hline Rabow et al., 2004 & Regularly & 8 & Not stated & Team Physician & Not stated & Care plan \\
\hline Yagura et al., 2005 & Weekly & 7 & Not stated & Not stated & Not stated & Discharge plan \\
\hline Gade et al., 2008 & Daily & 4 & Not stated & Not stated & Not stated & Palliative care plan \\
\hline Wolfs et al., 2009 & Weekly & 6 & Not stated & Not stated & Not stated & Care plan \\
\hline Hagiwara et al., 2011 & Twice per week & 4 & Not stated & Hemato-oncologist & Not stated & Care plan \\
\hline Pope et al., 2011 & Not stated & 3 & Not stated & Modified Delphi method & Not stated & Not stated \\
\hline
\end{tabular}

failure. One of them found that MDT care was costeffective with an incremental cost-effectiveness ratio of US\$1,068 per Quality Adjusted Life Year (QALY) ( $£ 663 /$ QALY, $£ 1=U S \$ 1.61$, rate as at 20 April 2012) [32]. However, the other RCT showed that there was no evidence of a real cost difference but quality of life improved when comparing MDT and no-MDT care [38].

Two RCTs evaluated MDT care amongst patients with terminal conditions; the results were mixed. One reported that costs were lower for the MDT group by about $30 \%$, but there was no evidence of any differences in measures of symptom control, survival, and quality of life between the MDT and no MDT groups [36]. In contrast, the other study showed that there was no evidence of a real cost difference but some outcome measures such as dyspnea, anxiety, spiritual well-being, improved when comparing MDT and no-MDT care [41].

The last three RCTs, each of people with a different disease, also showed varied results. One study relating to patients with mental health problems found that MDT care was cost-effective with an incremental cost effectiveness ratio of $€ 1,267 / \mathrm{QALY}(£ 1,039 / \mathrm{QALY}, £ 1=€ 1.22$, rate as at 20 April 2012) [45]. However, a RCT concerning stroke patients concluded that there was no evidence of real cost or outcome measures differences [46]. Furthermore, in a study that investigated the impact of MDT care versus no MDT care among patients diagnosed with rheumatoid arthritis, there was no evidence of differences in outcome measures but costs were higher in the MDT care group by approximately $30 \%$ to $50 \%$ [43].
Other study designs assessing cost-effectiveness of MDTs in non-cancer care

A prospective cohort study based in Sweden which considered the effect of MDT on vocational activity among patients with chronic minor diseases and long-term absence from working life, reported a total discounted cost of 7.2 million SEK $(£ 684,410, £ 1=10.52$ SEK, rate as at 20 April 2012), total benefits of 35.1 million SEK ( $£ 3.3$ million), and hence, a positive cost-benefit ratio of 4.9 [42]. No estimate for cost per person was provided. In addition, a 'before and after' study found that the rate of infection per 1000 patient days after the implementation of MDT antibiotic management care fell by between $25 \%$ to $70 \%$ and costs decreased by about $30 \%$ [33].

Studies assessing cost-effectiveness of MDTs in cancer care There were two non-randomised studies that reported results of MDTs in cancer care (Table 5). In a retrospective cohort study of MDT versus no MDT care for patients with malignant melanoma, the costs of health care were $33 \%$ to $50 \%$ lower in patients whose management decisions were made by an MDT [35]. However, there was no evidence of any differences in wound complication and 5-year survival rates. Hagiwara et al. (2011) [37] reported clinical outcomes before and after the introduction of a multidisciplinary nutritional team for patients with haematologic malignancies. It was found that incidences of hepatic complications, hyperglycemia, and central venous catheter infection were lower in the 'after' group than in the 'before' group, and costs fell by about $20 \%$. 


\begin{tabular}{|c|c|c|c|c|c|c|c|}
\hline $\begin{array}{l}\text { Authors, date, } \\
\text { and country }\end{array}$ & MDT team composition & $\begin{array}{l}\text { Patient group or } \\
\text { disease; sample size }\end{array}$ & $\begin{array}{l}\text { Study design; } \\
\text { type of economic } \\
\text { evaluation }\end{array}$ & $\begin{array}{l}\text { Comparison } \\
\text { group }\end{array}$ & $\begin{array}{l}\text { Outcome } \\
\text { measures }\end{array}$ & Costs included & Findings \\
\hline \multicolumn{8}{|c|}{ RCTs assessing cost-effectiveness of MDTs in non-cancer care } \\
\hline \multicolumn{8}{|l|}{ Geriatric care } \\
\hline \multirow[t]{4}{*}{$\begin{array}{l}\text { Collard et al., } \\
1985, \text { [34] USA }\end{array}$} & \multirow{4}{*}{$\begin{array}{l}\text { Primary nurse, social worker, } \\
\text { physician, physical therapist, } \\
\text { occupational therapist, } \\
\text { medical director }\end{array}$} & \multirow{4}{*}{$\begin{array}{l}\text { Geriatric patients; } \\
\text { treatment }=218 \\
\text { control = } 477\end{array}$} & \multirow{4}{*}{$\begin{array}{l}\mathrm{RCT} ; \\
\text { cost-consequences } \\
\text { analysis }\end{array}$} & \multirow{4}{*}{$\begin{array}{l}\text { Usual care } \\
\text { i.e. no MDT }\end{array}$} & \multirow{4}{*}{$\begin{array}{l}\text { Health status, complications } \\
\text { during hospitalisation, use } \\
\text { of physical/chemical } \\
\text { restraints at } 5 \text { months }\end{array}$} & \multirow{4}{*}{ Hospitalisation } & $\begin{array}{l}\text { Incremental costs of MDT - lower } \\
\text { by US\$564 (£350) per person }\end{array}$ \\
\hline & & & & & & & Morbidity -better for MDT group \\
\hline & & & & & & & Mortality - not measured \\
\hline & & & & & & & Cost per QALY: not calculated \\
\hline \multirow[t]{4}{*}{$\begin{array}{l}\text { Williams et al., } \\
\text { 1987, [44] USA }\end{array}$} & \multirow{4}{*}{$\begin{array}{l}\text { internists and family } \\
\text { physicians with special } \\
\text { expertise in geriatrics, } \\
\text { psychiatrists, nurses, social } \\
\text { workers, nutritionists }\end{array}$} & \multirow{4}{*}{$\begin{array}{l}\text { Geriatric patients; } \\
\text { treatment }=58 \\
\text { controls }=59\end{array}$} & \multirow{4}{*}{$\begin{array}{l}\mathrm{RCT} ; \\
\text { cost-consequences } \\
\text { analysis }\end{array}$} & \multirow{4}{*}{$\begin{array}{l}\text { Care by } 1 \\
\text { internist }\end{array}$} & \multirow{4}{*}{$\begin{array}{l}\text { Functional status, } \\
\text { institutional placement } \\
\text { at } 12 \text { months }\end{array}$} & \multirow{4}{*}{$\begin{array}{l}\text { Hospitalisation, nursing } \\
\text { home, home aid, } \\
\text { transportation, GP visit, } \\
\text { day centre, visits by various } \\
\text { health professionals, } \\
\text { meals-on-wheels, nurse \& } \\
\text { homemaker hours }\end{array}$} & $\begin{array}{l}\text { Incremental costs of } \\
\text { MDT - equivalent }\end{array}$ \\
\hline & & & & & & & Morbidity - equivalent \\
\hline & & & & & & & Mortality - not measured \\
\hline & & & & & & & Cost per QALY: not calculated \\
\hline \multirow[t]{4}{*}{$\begin{array}{l}\text { Kominski et al., } \\
\text { 2001, [39] USA }\end{array}$} & \multirow{4}{*}{$\begin{array}{l}\text { Nurses, psychiatrists, } \\
\text { psychologists, social } \\
\text { workers, geriatricians, } \\
\text { nutritionists, pharmacists }\end{array}$} & \multirow{4}{*}{$\begin{array}{l}\text { Geriatric patients; } \\
\text { treatment }=814 \\
\text { usual care }=873\end{array}$} & \multirow{4}{*}{$\begin{array}{l}\text { RCT; } \\
\text { cost-consequences } \\
\text { analysis }\end{array}$} & \multirow{4}{*}{$\begin{array}{l}\text { Usual care } \\
\text { i.e. no MDT }\end{array}$} & \multirow{4}{*}{$\begin{array}{l}\text { 36-item Health Survey } \\
\text { Short Form (SF-36), } \\
\text { Mental Health Inventory } \\
\text { (MHI) at } 12 \text { months }\end{array}$} & \multirow[t]{4}{*}{$\begin{array}{l}\text { Inpatient, ambulatory } \\
\text { care clinic }\end{array}$} & $\begin{array}{l}\text { Incremental costs of } \\
\text { MDT - equivalent }\end{array}$ \\
\hline & & & & & & & Morbidity - equivalent \\
\hline & & & & & & & Mortality - not measured \\
\hline & & & & & & & Cost per QALY: not calculated \\
\hline \multirow{4}{*}{$\begin{array}{l}\text { Pope et al., } \\
\text { 2011, [40] } \\
\text { Ireland }\end{array}$} & \multirow{4}{*}{$\begin{array}{l}\text { Consultant geriatrician, } \\
\text { specialist registrar in } \\
\text { geriatric medicine, } \\
\text { pharmacists, nurse } \\
\text { practitioners }\end{array}$} & \multirow{4}{*}{$\begin{array}{l}\text { Geriatric patients; } \\
\text { treatment }=110, \\
\text { control = } 115\end{array}$} & \multirow{4}{*}{$\begin{array}{l}\text { RCT; } \\
\text { cost-consequences } \\
\text { analysis }\end{array}$} & \multirow{4}{*}{$\begin{array}{l}\text { Regular } \\
\text { assessment } \\
\text { i.e. no MDT }\end{array}$} & \multirow{4}{*}{$\begin{array}{l}\text { Mortality, Barthel Index, } \\
\text { Abbreviated Mental } \\
\text { Test Score (AMTS) } \\
\text { at } 6 \text { months }\end{array}$} & \multirow[t]{4}{*}{$\begin{array}{l}\text { Medical review, medication, } \\
\text { acute hospital transfer }\end{array}$} & $\begin{array}{l}\text { Incremental costs of } \\
\text { MDT - higher by } £ 510 \text { per person }\end{array}$ \\
\hline & & & & & & & Morbidity - equivalent \\
\hline & & & & & & & Mortality - equivalent \\
\hline & & & & & & & Cost per QALY: not calculated \\
\hline \multicolumn{8}{|l|}{ Heart failure care } \\
\hline \multirow[t]{4}{*}{$\begin{array}{l}\text { Capomolla } \\
\text { et al., 2002, [32] } \\
\text { Italy }\end{array}$} & $\begin{array}{l}\text { cardiologist, nurses, } \\
\text { physiotherapists; part-time } \\
\text { participation of dietician, }\end{array}$ & $\begin{array}{l}\text { Heart failure; } \\
\text { treatment }=112 \\
\text { control }=122\end{array}$ & $\begin{array}{l}\mathrm{RCT} \text {; cost-utility } \\
\text { analysis }\end{array}$ & $\begin{array}{l}\text { Usual care by } \\
\text { cardiologist }\end{array}$ & $\begin{array}{l}\text { Death, QALY at } \\
12 \text { months }\end{array}$ & $\begin{array}{l}\text { Pharmacologic, care } \\
\text { management }\end{array}$ & $\begin{array}{l}\text { Incremental costs of } \\
\text { MDT - lower by US } \$ 10,768 \\
(£ 6,688) \text { per person }\end{array}$ \\
\hline & & & & & & & $\begin{array}{l}\text { Morbidity - better for the } \\
\text { MDT group }\end{array}$ \\
\hline & & & & & & & Mortality - lower for MDT group \\
\hline & & & & & & & Cost per QALY: US\$1,068 (£663) \\
\hline $\begin{array}{l}\text { Kasper et al., } \\
\text { 2002, [38] USA }\end{array}$ & $\begin{array}{l}\text { Telephone nurse co-ordinator, } \\
\text { CHF nurse, CHF cardiologist, }\end{array}$ & $\begin{array}{l}\text { heart failure; } \\
\text { treatment = 102, }\end{array}$ & $\begin{array}{l}\text { RCT; cost- } \\
\text { consequences }\end{array}$ & $\begin{array}{l}\text { Care provided } \\
\text { by GP only }\end{array}$ & $\begin{array}{l}\text { Death, quality of life } \\
\text { (QoL) at } 6 \text { months }\end{array}$ & $\begin{array}{l}\text { Personnel, inpatient, } \\
\text { outpatient pharmacy, }\end{array}$ & $\begin{array}{l}\text { Incremental costs of } \\
\text { MDT - equivalent }\end{array}$ \\
\hline & & & & & & & $\begin{array}{l}\text { Morbidity - better for the } \\
\text { MDT group }\end{array}$ \\
\hline
\end{tabular}


Rheumatoid arthritis care, stroke care, dementia care

$\begin{array}{llll}\text { Van den Hout } & \text { Nurse, rheumatologist } & \text { Rheumatoid arthritis; } & \text { RCT; cost- } \\ \text { et al., 2003, } & \text { occupational therapist, } & \text { treatment }=71 & \text { consequences } \\ \text { [43] The } & \text { physical therapist, social } & \text { (inpatient MDT), } & \text { analysis } \\ \text { Netherlands } & \text { worker. } & 68 \text { (outpatient MDT) } & \\ & & \text { control }=71 & \end{array}$

Usual care Functional status, quality i.e. no MDT of life at $6,12,26,52$, and 104 weeks

Terminal/critical care

Rabow et al. Social worker, nurse, chaplain, Life limiting diseass 2004, [41] USA pharmacist, psychologist, art $\begin{array}{lll}\text { pharmacist, psychologist, art } & \text { such as cancer, } & \text { consequenc } \\ \text { therapist, volunteer } & \text { advanced COPD, or } & \text { analysis } \\ \text { coordinator, physician } & \text { advanced CHF; } & \end{array}$ treatment $=50$

Gade et al., Palliative care physician,

A range of life-limiting $\mathrm{RCT}$; cost-

2008, [36] USA nurse, hospital social worker and chaplain $\begin{array}{ll}\text { diseases such as } & \text { consequenc } \\ \text { COPD, stroke, cancer; } & \text { analysis }\end{array}$ treatment $=280$

Usual care i.e no MDT

Symptom severity, quality of life and
6 months

Hospitalisation, pharmacologic, study

Mortality - equivalent

Cost per QALY: not calculated

MDT - equivalent

Morbidity - better for MDT group

Mortality - not calculated

Cost per QALY: not calculated

ncremental costs of

MDT - lower by US\$4,855

$£ 3,016)$ per patient

Morbidity - equivalent

Mortality - equivalent

Cost per QALY: not calculated

Hospitalisations, personnel, Incremental costs of

MDT -higher by $€ 5,160$ to $€ 10,876$

home nursing care, other

health professionals drugs,

and appliances, out of

$£ 4,230$ to $£ 8,915)$ per person

are informal

care, paid and unpaid labour

Morbidity - equivalent

Mortality - not measured

Usual care Functional status, Hospitalisation

Yagura et al.,

Physicians, nurses, physical

Stroke; treatment $=91, \mathrm{RCT}$; cost -

therapists, occupational
therapists, speech therapists,

control $=87 \quad$ consequences

Japan

clinical psychologists, social

analysis

no MDT impairment status

(duration of measurement not stated)

worker

Usual care

QALYS, cognition

Wolfs et al.,

Old age psychiatry, geriatric Patients suspected of RCT; cost-utility 2009, [45] The medicine, neuropsychology, having dementia or a analysis physiotherapy, occupational cognitive disorder;

therapy, geriatric nursing and treatment $=13$

mental health nursing control $=88$

problems at 6 months and 12 months

Medical, informal care,

Cost per QALY: not calculated

Incremental costs of

MDT -equivalent

Morbidity - equivalent

Mortality - not measured

Cost per QALY: not calculated

Incremental costs of MDT - higher by €65 (£53) per person

Morbidity - better for MDT group

Mortality - not measured

Cost per QALY: $€ 1,267(£ 1,039)$

\section{Other study designs assessing cost-effectiveness of MDTs in non-cancer care}

$\begin{array}{llll}\begin{array}{l}\text { Timpka et al., } \\ \text { 1997, [42] }\end{array} & \begin{array}{l}\text { Part-time physicians, } \\ \text { psychologist, social workers }\end{array} & \begin{array}{l}\text { Patients with chronic } \\ \text { minor diseases and } \\ \text { long-term absence } \\ \text { lonort; cost- } \\ \text { from working life; } 239\end{array}\end{array}$

baseline Vocational activity,

characteristics benefits to society

before start of at 12 months and

programme 5 years

Programme, indirect

Costs - 30,000 SEK $(£ 2,852)$

per person

Benefits - 1.25 million

SEK $(£ 117,500)$ per person

Cost-benefit ratio -4.9 
Table 5 Details of included studies $(n=15)$ (Continued)

\begin{tabular}{|c|c|c|c|c|c|c|c|}
\hline \multirow[t]{4}{*}{$\begin{array}{l}\text { Carling et al., } \\
\text { 2003, [33] USA }\end{array}$} & \multirow[t]{4}{*}{$\begin{array}{l}\text { Clinical pharmacist, infectious } \\
\text { diseases physician }\end{array}$} & \multirow{4}{*}{$\begin{array}{l}\text { Adults receiving } \\
\text { parenteral 3rd } \\
\text { generation } \\
\text { cephalosporins, } \\
\text { aztreonam, parenteral } \\
\text { fluoroquinolones, or } \\
\text { imipenem; sample size } \\
\text { not specified }\end{array}$} & \multirow[t]{4}{*}{$\begin{array}{l}\mathrm{B} \& \mathrm{~A} ; \text { cost- } \\
\text { consequences } \\
\text { analysis }\end{array}$} & \multirow[t]{4}{*}{$\begin{array}{l}\text { Before MDT } \\
\text { implemented }\end{array}$} & \multirow[t]{4}{*}{$\begin{array}{l}\text { Incidence of } \\
\text { nosocomial } \\
\text { infections per } 1000 \\
\text { patient-days }\end{array}$} & \multirow[t]{4}{*}{ Medication } & $\begin{array}{l}\text { Incremental costs of } \\
\text { MDT - lower by } \\
\text { US\$200,000 to US } \$ 250,000 \\
\text { per year ( } £ 124,224 \text { to } £ 155,280)\end{array}$ \\
\hline & & & & & & & Morbidity - better for MDT group \\
\hline & & & & & & & Mortality - not measured \\
\hline & & & & & & & Cost per QALY: not calculated \\
\hline \multicolumn{8}{|c|}{$\underline{\text { Studies assessing cost-effectiveness of MDT in cancer care }}$} \\
\hline \multirow[t]{4}{*}{$\begin{array}{l}\text { Fader et al., } \\
\text { 1998, [35] USA }\end{array}$} & \multirow{4}{*}{$\begin{array}{l}\text { specialists in dermatology; } \\
\text { surgical, medical, \& radiation } \\
\text { oncology; plastic \& } \\
\text { dermatologic surgery; } \\
\text { otorhinolaryngology; } \\
\text { obstetrics/gynecology; } \\
\text { ophthalmology, } \\
\text { dermatopathology; nuclear } \\
\text { medicine; and social work }\end{array}$} & \multirow[t]{4}{*}{$\begin{array}{l}\text { Melanoma; } \\
\text { treatment }=104 \\
\text { control }=104\end{array}$} & \multirow[t]{4}{*}{$\begin{array}{l}\text { Cohort; } \\
\text { cost-consequences } \\
\text { analysis }\end{array}$} & \multirow[t]{4}{*}{$\begin{array}{l}\text { Usual care } \\
\text { i.e. no MDT }\end{array}$} & \multirow[t]{4}{*}{$\begin{array}{l}\text { Surgical morbidity } \\
\text { at } 1 \text { month, survival } \\
\text { at } 5 \text { years }\end{array}$} & \multirow[t]{4}{*}{$\begin{array}{l}\text { Diagnosis and initial } \\
\text { management }\end{array}$} & $\begin{array}{l}\text { Incremental costs of } \\
\text { MDT - lower by US } \\
\$ 1,595 \text { (£991) per person }\end{array}$ \\
\hline & & & & & & & Morbidity - equivalent \\
\hline & & & & & & & Mortality - equivalent \\
\hline & & & & & & & Cost per QALY: not calculated \\
\hline \multirow[t]{4}{*}{$\begin{array}{l}\text { Hagiwara et al., } \\
\text { 2011, [37] } \\
\text { Japan }\end{array}$} & \multirow[t]{4}{*}{$\begin{array}{l}\text { Hemato-oncologist, nurse, } \\
\text { dietitian, pharmacist }\end{array}$} & \multirow{4}{*}{$\begin{array}{l}\text { Hematologic } \\
\text { malignancies; } \\
\text { Before - 67, } \\
\text { After - } 102\end{array}$} & \multirow[t]{4}{*}{$\begin{array}{l}\text { B\&A; } \\
\text { cost-consequences } \\
\text { analysis }\end{array}$} & \multirow[t]{4}{*}{ No MDT } & \multirow{4}{*}{$\begin{array}{l}\text { Number of } \\
\text { adverse events, } \\
\text { death (duration } \\
\text { of measurement } \\
\text { not stated) }\end{array}$} & \multirow{4}{*}{$\begin{array}{l}\text { Parenteral nutrition, } \\
\text { antibiotics, food and } \\
\text { nutritional supplement, } \\
\text { MDT personnel }\end{array}$} & $\begin{array}{l}\text { Incremental costs of } \\
\text { MDT - lower by } 403,600 \\
\text { yen }(£ 3,058) \text { per person }\end{array}$ \\
\hline & & & & & & & Morbidity - better for MDT group \\
\hline & & & & & & & Mortality - equivalent \\
\hline & & & & & & & Cost per QALY: not calculated \\
\hline
\end{tabular}




\section{Discussion}

\section{Summary of findings}

We identified fifteen primary studies on the costeffectiveness of MDT decision making in secondary care, of which only two concerned cancer and the others covered a wide range of disease types. The characteristics of MDT meetings were diverse. The existing evidence was limited and there was a relatively high risk of bias amongst the studies. Whilst both studies relating to cancer care showed that MDTs were associated with lower costs and better outcomes or outcomes comparable to no MDT care, neither study was a RCT. The results from eleven RCTs assessing the cost-effectiveness of MDTs in non-cancer care were very varied. Only three of these showed that MDT working in secondary care had a positive effect on outcomes and costs. However, the degree of this positive impact varied. Moreover, the positive impact of MDT on some costs components might be offset by the full cost of administering, preparing for and attending an MDT meeting, for which no estimates were reported in the included studies. Therefore, based on existing evidence, it is not possible to determine if MDT working is cost-effective or not in secondary cancer and non-cancer care.

\section{Strengths and weaknesses of this systematic review}

To our knowledge, we conducted the first systematic review on the cost-effectiveness of MDT working in secondary care, and we explicitly attempted to capture all types of costs, especially the significant costs of supporting MDT working. The limitations of this review need to be borne in mind. Firstly, as with any systematic review, publication bias might be a problem if studies with null findings have not been published. Secondly, this review was not restricted to a particular country or disease type. While this approach is valuable in describing the available evidence from a wide perspective, it restricts the comparability across studies. Given that the studies were diverse, particularly in the type of care offered in the comparison groups, the findings may not be applicable across different healthcare settings where different clinical practices and geographical constraints operate. The operationalising of MDT may also differ in different settings and together with the variability in reporting, there may be studies that have been conceived as MDT, but based on our interpretation, were not. However, we tried to be inclusive in our definition of MDT working so that we did not exclude potentially relevant studies.

\section{Weaknesses of the evidence}

We have identified four key weaknesses in the existing literature on the cost-effectiveness of MDT working. First, there was no standard definition or operationalisation of MDT working evident in the literature. Most studies did not provide key details of the MDT meeting venue, leadership (i.e. who leads the meeting and or how are decisions made), or administrative support. Without a common definition or clear description of MDTs, it was difficult to compare results between studies or draw conclusions on the cost-effectiveness of MDT decision making. Second, the full costs of administering, preparing for and attending an MDT meeting were not estimated in the included studies. Given that the MDT meeting is a key feature of MDT working, the omission of the associated costs in any cost-effectiveness analysis of MDT could lead to an underestimation of costs, and hence bias in the results. Third, the methodological quality assessment has demonstrated that there was a relatively high risk of bias amongst the studies. This meant that the reliability and reproducibility of the included studies were compromised, and the ability to determine conclusions from our systematic review was restricted. Fourth, the available evidence was limited so that it was not possible to draw firm conclusions as to whether MDT working in cancer or non-cancer care would lead to cost-effective care or not.

\section{Comparison with other studies}

In their review to determine the critical elements of effective team-working in patient care teams, Bosch et al. [47] concluded that current literature provided little insight into the underlying mechanisms. They reported results relating to costs and resource utilisation but not on cost-effectiveness of MDTs. In comparison, our systematic review focused specifically on full economic evaluations of MDT working, and sought to include studies that estimated the costs of supporting MDT working. In another review examining the association between cancer MDTs and survival, the authors also highlighted the lack of a consistent definition of MDT [21]. This directly hampers any investigation of its effectiveness and costs. While none of the included studies in this review had accounted for the full costs of administering, preparing for and attending an MDT meeting, an audit conducted at a large teaching hospital/cancer centre estimated the average annual cost of MDTs in salaries alone was about $€ 1$ million [48]. Time spent preparing for MDT meetings could be considerable. A study examining the participation of pathologists and radiologists in MDT meetings found that the former spent about an average of 2.4 hours, and the latter 2 hours in preparation [49]. Although our review did not find evidence that MDT working is cost-effective in cancer care, there appears to be a growing consensus that telemedicine delivered MDT cancer meetings are more cost-effective than 'in-person' meetings [50-53].

\section{Future research}

Based on the findings of our systematic review, we propose that three issues need to be addressed in future 
research in this area. Firstly, future research should ensure a clear definition of MDT working and detailed reporting of its characteristics. In our systematic review, we found that the description of the two key features of MDT working was so vague that it was difficult to determine whether MDT working had taken place or not. We recommend that studies should provide the characteristics of the MDT meeting as shown in Table 4. Secondly, it is essential to estimate the full costs of administering, preparing for and attending an MDT meeting. Such attempts should take into account two issues. One, all relevant costs should be identified. We recommend that the following costs should be accounted for: time spent by each team member in preparing for the MDT meeting and this should include time spent by team coordinator in providing administrative support to the MDT meeting, time spent on travelling to and from meetings by any team member who is located on a different site than the meeting venue, costs of using teleconferencing if applicable, and time spent by each member at each meeting. Two, these costs should be measured as accurately as possible. The standard methods used in health services research for workflow assessments are work sampling, time efficiency, and time-and-motion. The first two methods are less resource intensive as compared to the third method but there is evidence to show that the time-and-motion technique may produce the most accurate results [54-56]. Thirdly, it remains unclear what models of MDT are cost-effective in secondary care. Therefore, future studies using strong study designs should compare the cost-effectiveness of different models or aspects of MDT working ${ }^{\mathrm{c}}$. For this to be performed accurately there is a need for agreement in the clinical community about which outcomes would be core in the evaluation of MDT working. Whilst some clinical outcomes are important, the inclusion of patient-centred outcomes and satisfaction of health professionals as well as assessment of the quality of decision-making and MDT decision-implementation may also be important $[2,57,58]$.

\section{Policy implications}

Without further research that addresses the three key issues highlighted in the previous section, it would be hard to make firm policy recommendations. The costeffectiveness of MDT working in secondary care is likely to vary according to many factors, such as cost of supporting MDT working, disease type, treatment selected, and geographical location. The costs of supporting MDT working are directly linked to factors that affect management decisions of MDTs, such as inclusion of time to prepare for MDTs into team-members' job plans, making team and leadership skills training available to team-members, and systematic input from nursing personnel [59]. The commitment of resources to these factors will differ across settings. Therefore, it is likely that there is no 'one-size-fits-all' model of MDT working to suit all diseases, treatment modalities and locations.

\section{Conclusion}

The widespread adoption of MDT working in cancer and non-cancer care has occurred despite a lack of evidence on cost-effectiveness. The available evidence is limited and at relatively high risk of bias. It is not possible to determine if MDT working in cancer and non-cancer care is cost-effective or not. Further studies aimed at understanding the key aspects of MDT working that lead to cost-effective care, and the costs of supporting MDT working are required.

\section{Endnotes}

${ }^{a}$ Costs can be derived by a bottom-up or top-down approach. In a bottom-up approach, costs are determined by examining individual utilisation. In a top-down approach, costs are apportioned across individuals, and this may be less accurate due to questionable assumptions used in the apportionment of costs.

${ }^{\mathrm{b}}$ Personal communication with the lead author has confirmed that these costs did not include the full costs of administering, preparing for and attending an MDT meeting.

${ }^{c}$ As MDT working is now so prevalent in secondary care, especially cancer care, it might not be possible to compare the cost-effectiveness of no-MDT versus MDT working.

\section{Appendix 1}

Search Strategy

EMBASE

\#1. multidisciplinary OR "multidisciplinary team" OR interdisciplinary OR "interdisciplinary team" OR team" OR "patient care team" OR "health care team" "medical care team"

\#2. "cost savings" OR "cost-effective" OR "cost-utility" OR "economic evaluation" OR economic" OR "cost analysis" OR "health care costs" \#3. hospital or "hospital setting" OR "hospital based" OR "secondary care"

\#4. \#1 AND \#2 AND \#3

\#5. limit to (human and English language) AND (article) MEDLINE (MeSH terms used)

\#1. (Patient Care Team)

\#2. ((Costs and Cost Analysis) OR (Economic*) OR (Cost-Benefit Analysis) OR (Cost Savings) OR (Health Care Costs) OR (Cost of Illness) OR (Health Services Accessibility) OR (Patient Transfer) OR (Travel) OR (Transportation of Patients)

\#3. \#1 AND \#2

\#4. limit \#3 to (English language and humans)

NHS EED 
(multidisciplinary OR "multidisciplinary team" interdisciplinary OR "interdisciplinary health team" OR team" OR "patient care team" OR "health care team" OR "medical care team"”) (english:la)

CINAHL

\#1. multidisciplinary OR "multidisciplinary team" OR interdisciplinary OR "interdisciplinary health team" ${ }^{*}$ " OR team" OR "patient care team" ${ }^{*}$ OR "health care team" OR "medical care team"

\#2. "cost savings" OR "cost-effective"*" OR "cost-benefit" OR "cost-utility" OR "economic evaluation" OR economic" OR "cost analysis" OR 'health care costs' OR access" $^{*}$ OR travel OR burden

\#3. \#1 AND \#2

\#4. limit to English Language; Peer Reviewed; Research Article; Exclude MEDLINE records.

EconLit

\#1. multidisciplinary OR "multidisciplinary team" OR interdisciplinary OR "interdisciplinary health team" OR team* OR "patient care team" OR "health care team*" OR "medical care team" "

\#2. "cost savings" OR "cost-effective" OR "cost-utility" OR "economic evaluation" OR economic" OR "cost analysis" OR 'health care costs' OR access" OR travel OR burden

\#3. \#1 AND \#2

\#4 limit to (English language) AND (article or conference paper)

Cochrane Library

multidisciplinary OR "multidisciplinary team"” OR interdisciplinary OR "interdisciplinary health team" " OR team" OR "patient care team" OR "health care team"” OR "medical care team"" in Title, Abstract or Keyword and "cost savings" OR "cost-effective" $e^{*}$ OR "cost-benefit" OR "costutility" OR "economic evaluation" OR economic" OR "cost analysis" OR 'health care costs' OR access" OR travel OR burden in in Title, Abstract or Keyword

NHS HMIC

(multidisciplinary OR "multidisciplinary team" OR interdisciplinary OR "interdisciplinary health team" OR team* OR "patient care team*" OR "health care team*" OR "medical care team"*) (english:la)

\section{Competing interests}

The authors declare that they have no competing interests.

\section{Authors' contributions}

KMK, JMB, ARN, WH conceived of the study and participated in its design. All authors analysed the data, drafted the manuscript, and read and approved the final manuscript.

\section{Acknowledgements}

This paper presents independent research funded by the National Institute for Health Research (NIHR) under its Programme Grants for Applied Research Programme (Grant Reference Number RP-PG-0707-10034). The views expressed are those of the authors and not necessarily those of the NHS, the NIHR or the Department of Health.

\section{Author details}

'School of Oral and Dental Sciences, University of Bristol, Lower Maudlin Street, Bristol BS1 2LY, UK. ${ }^{2}$ School of Social and Community Medicine, University of Bristol, Canynge Hall, 39 Whatley Road, Bristol BS8 2PS, UK. ${ }^{3}$ University Hospitals Bristol NHS Foundation Trust, Dolphin House, Bristol Royal Infirmary, Bristol BS2 8HW, UK.

Received: 19 September 2012 Accepted: 12 March 2013

Published: 4 April 2013

\section{References}

1. Fleissig A, et al: Multidisciplinary teams in cancer care: are they effective in the UK? Lancet Oncol 2006, 7(11):935-943.

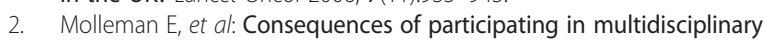
medical team meetings for surgical, nonsurgical, and supporting specialties. Med Care Res Rev 2010, 67(2):173-193.

3. Lemieux-Charles L, McGuire WL: What do we know about health care team effectiveness? A review of the literature. Med Care Res Rev 2006, 63(3):263-300

4. Rodriguez HP, et al: The effect of care team composition on the quality of HIV care. Med Care Res Rev 2008, 65(1):88-113.

5. Baker DP, Day R, Salas E: Teamwork as an essential component of highreliability organizations. Health Serv Res 2006, 41 (4 Pt 2):1576-1598.

6. Department of Health: The NHS cancer plan: a plan for investment, a plan for reform. London: Department of Health; 2000.

7. Department of Health: The NHS plan: a plan for investment, a plan for reform. London: Department of Health; 2000.

8. Institute of Medicine: Crossing the quality chasm: a new health system for the 21st century. Washington D.C: National Academies Press; 2001.

9. Wagner $\mathrm{EH}$ : The role of patient care teams in chronic disease management. BMJ 2000, 320(7234):569-572.

10. Tripathy D: Multidisciplinary care for breast cancer: barriers and solutions. Breast J 2003, 9(1):60-63.

11. Luxford K, Rainbird K: Multidisciplinary care for women with breast cancer: a national demonstration program. N S W Public Health Bull 2001, 12(10):277-279.

12. Zorbas $\mathrm{H}$, et al: Multidisciplinary care for women with early breast cancer in the Australian context: what does it mean? Med J Aust 2003, 179(10):528-531.

13. Magnani T, et al: The 6-year attendance of a multidisciplinary prostate cancer clinic in Italy: incidence of management changes. BJU Int 2012, 110(7):998-1003.

14. van Nes J, an de Velde C: The multidisciplinary breast cancer team: promoting better care. Ned Tijdschr Geneeskd 2005, 149:1929-1931.

15. Gabel M, Hilton NE, Nathanson SD: Multidisciplinary breast cancer clinics. Do they work? Cancer 1997, 79(12):2380-2384.

16. Davies $A R$, et al: The multidisciplinary team meeting improves staging accuracy and treatment selection for gastro-esophageal cancer. Dis Esophagus 2006, 19(6):496-503.

17. Junor EJ, Hole DJ, Gillis CR: Management of ovarian cancer: referral to a multidisciplinary team matters. Br J Cancer 1994, 70(2):363-370.

18. Kingsmore $D$, Hole $D$, Gillis $C$ : Why does specialist treatment of breast cancer improve survival? The role of surgical management. $\mathrm{Br} J$ Cancer 2004, 90(10):1920-1925.

19. Kesson EM, et al: Effects of multidisciplinary team working on breast cancer survival: retrospective, comparative, interventional cohort study of 13722 women. BMJ 2012, 344:e2718.

20. Boxer $\mathrm{MM}$, et al: Do multidisciplinary team meetings make a difference in the management of lung cancer? Cancer 2011, 117(22):5112-5120.

21. Hong NJ, et al: Examining the potential relationship between multidisciplinary cancer care and patient survival: an international literature review. J Surg Oncol 2010, 102(2):125-134.

22. Tattersall MH: Multidisciplinary team meetings: where is the value? Lancet Oncol 2006, 7(11):886-888.

23. Taylor C, et al: Multidisciplinary team working in cancer: what is the evidence? BMJ 2010, 340:c951.

24. Department of Health: Guidance on commissioning cancer services: Improving outcomes in upper gastro-intestinal cancers. London: Department of Health; 2001.

25. McNair AG, et al: Maximising recruitment into randomised controlled trials: the role of multidisciplinary cancer teams. Eur J Cancer 2008, 44(17):2623-2626. 
26. Goolam-Hossen T, et al: Waiting times for cancer treatment: the impact of multi-disciplinary team meetings. Behaviour \& Information Technology 2011, 30(4):467-471.

27. NSW Department of Health: Multidisciplinary ward rounds: A resource. Sydney: NSW Department of Health; 2011.

28. Drummond MF, et al: Methods for the economic evaluation of health care programmes. 3rd edition. Oxford: Oxford University Press; 2005.

29. Centre for Reviews and Dissemination: Systematic reviews: CRD's guidance for undertaking reviews in health care. York: CRD, University of York; 2009.

30. Evers $\mathrm{S}$, et al: Criteria list for assessment of methodological quality of economic evaluations: Consensus on Health Economic Criteria. Int J Technol Assess Health Care 2005, 21(2):240-245.

31. Uegaki $\mathrm{K}$, et al: Economic evaluations of occupational health interventions from a corporate perspective - a systematic review of methodological quality. Scand J Work Environ Health 2010, 36(4):273-288.

32. Capomolla S, et al: Cost/utility ratio in chronic heart failure: comparison between heart failure management program delivered by day-hospital and usual care. J Am Coll Cardiol 2002, 40(7):1259-1266.

33. Carling $P$, et al: Favorable impact of a multidisciplinary antibiotic management program conducted during 7 years. Infect Control Hosp Epidemiol 2003, 24(9):699-706.

34. Collard AF, Bachman SS, Beatrice DF: Acute care delivery for the geriatric patient: an innovative approach. QRB Qual Rev Bull 1985, 11(6):180-185.

35. Fader DJ, et al: The multidisciplinary melanoma clinic: a cost outcomes analysis of specialty care. J Am Acad Dermatol 1998, 38(5 Pt 1):742-751.

36. Gade $G$, et al: Impact of an inpatient palliative care team: a randomized control trial. J Palliat Med 2008, 11(2):180-190.

37. Hagiwara $S$, et al: Multidisciplinary nutritional support for autologous hematopoietic stem cell transplantation: a cost-benefit analysis. Nutrition 2011, 27(11-12):1112-1117.

38. Kasper EK, et al: A randomized trial of the efficacy of multidisciplinary care in heart failure outpatients at high risk of hospital readmission. J Am Coll Cardiol 2002, 39(3):471-480.

39. Kominski $\mathrm{G}$, et al: UPBEAT: the impact of a psychogeriatric intervention in VA medical centers. Unified Psychogeriatric Biopsychosocial Evaluation and Treatment. Med Care 2001, 39(5):500-512.

40. Pope $\mathrm{G}$, et al: Specialist medication review does not benefit short-term outcomes and net costs in continuing-care patients. Age Ageing 2011, 40(3):307-312

41. Rabow MW, et al: The comprehensive care team: a controlled trial of outpatient palliative medicine consultation. Arch Intern Med 2004, 164(1):83-91.

42. Timpka T, et al: Long-term economic effects of team-based clinical case management of patients with chronic minor disease and long-term absence from working life. Scand J Soc Med 1997, 25(4):229-237.

43. van den Hout WB, et al: Cost effectiveness and cost utility analysis of multidisciplinary care in patients with rheumatoid arthritis: a randomised comparison of clinical nurse specialist care, inpatient team care, and day patient team care. Ann Rheum Dis 2003, 62(4):308-315.

44. Williams ME, et al: How does the team approach to outpatient geriatric evaluation compare with traditional care: a report of a randomized controlled trial. J Am Geriatr Soc 1987, 35(12):1071-1078.

45. Wolfs CA, et al: Economic evaluation of an integrated diagnostic approach for psychogeriatric patients: results of a randomized controlled trial. Arch Gen Psychiatry 2009, 66(3):313-323.

46. Yagura $\mathrm{H}$, et al: Patients with severe stroke benefit most by interdisciplinary rehabilitation team approach. Cerebrovasc Dis 2005, 20(4):258-263.

47. Bosch $M$, et al: Effectiveness of patient care teams and the role of clinical expertise and coordination: a literature review. Med Care Res Rev 2009, 66(6 Suppl):5S-35S.

48. Fosker CJ, Dodwell D: The cost of the MDT [Rapid Response]. 2010. http://www. bmj.com/rapid-response/2011/11/02/cost-mdt. accessed on 23 August 2012.

49. Kane $B$, et al: Multidisciplinary team meetings and their impact on workflow in radiology and pathology departments. BMC Med 2007, 5:15.

50. Kitamura C, Zurawel-Balaura L, Wong RK: How effective is video consultation in clinical oncology? A systematic review. Curr Oncol 2010, 17(3):17-27.

51. Kunkler $\mid \mathrm{H}$, et al: TELEMAM: a cluster randomised trial to assess the use of telemedicine in multi-disciplinary breast cancer decision making. Eur J Cancer 2007, 43(17):2506-2514.
52. Stalfors J, Bjorholt I, Westin T: A cost analysis of participation via personal attendance versus telemedicine at a head and neck oncology multidisciplinary team meeting. J Telemed Telecare 2005, 11(4):205-210.

53. Westin T, Stalfors J: Tumour boards/multidisciplinary head and neck cancer meetings: are they of value to patients, treating staff or a political additional drain on healthcare resources? Curr Opin Otolaryngol Head Neck Surg 2008, 16(2):103-107.

54. Ampt A, et al: A comparison of self-reported and observational work sampling techniques for measuring time in nursing tasks. $J$ Health Serv Res Policy 2007, 12(1):18-24.

55. Burke TA, et al: A comparison of time-and-motion and self-reporting methods of work measurement. J Nurs Adm 2000, 30(3):118-125.

56. Finkler SA, et al: A comparison of work-sampling and time-and-motion techniques for studies in health services research. Health Serv Res 1993, 28(5):577-597.

57. Blazeby JM, et al: Analysis of clinical decision-making in multi-disciplinary cancer teams. Ann Oncol 2006, 17(3):457-460.

58. Nallamothu BK, Cohen DJ: No "i" in Heart Team: incentivizing multidisciplinary care in cardiovascular medicine. Circ Cardiovasc Qual Outcomes 2012, 5(3):410-413.

59. Lamb BW, et al: Quality of care management decisions by multidisciplinary cancer teams: a systematic review. Ann Surg Oncol 2011, 18(8):2116-2125.

doi:10.1186/1478-7547-11-7

Cite this article as: Ke et al:: Are multidisciplinary teams in secondary care cost-effective? A systematic review of the literature. Cost Effectiveness and Resource Allocation 2013 11:7.

\section{Submit your next manuscript to BioMed Central and take full advantage of:}

- Convenient online submission

- Thorough peer review

- No space constraints or color figure charges

- Immediate publication on acceptance

- Inclusion in PubMed, CAS, Scopus and Google Scholar

- Research which is freely available for redistribution

Submit your manuscript at www.biomedcentral.com/submit
C) Biomed Central 